\title{
A Mother and Daughter with Unexplained Renal Failure
}

\author{
R.E. Christiansen ${ }^{a}$ T. Fiskerstrand ${ }^{b}$ S. Leh ${ }^{c}$ B.I. Haukanes ${ }^{b}$ A.K. Singh ${ }^{d}$ \\ F.C. Fervenza ${ }^{\text {e }}$ E. Svarstad ${ }^{a}, f$ \\ ${ }^{a}$ Department of Medicine, ${ }^{b}$ Center for Medical Genetics and Molecular Medicine, and ${ }^{\mathrm{c} D e p a r t m e n t}$ of \\ Pathology, Haukeland University Hospital, Bergen, Norway; ${ }^{d}$ Renal Division, Brigham and Women's Hospital, \\ Harvard Medical School, Boston, Mass., and ' Division of Nephrology and Hypertension, Mayo Clinic, Rochester, \\ Minn., USA; Institute of Medicine, University of Bergen, Bergen, Norway
}

\section{Biography}

Einar Svarstad received his MD from the University of Bergen in 1974, and today is Professor of Medicine at the same university as well as Head of the Renal Unit at Haukeland University Hospital. He has published numerous articles in peer-review journals and has been a member of several committees, such as the Norwegian Society of Nephrology and Scandinavian Society of Nephrology.

\section{Case Presentation}

Dr. Christiansen: Patient 1 (the mother) was referred to the nephrology department in 1991 at the age of 45 . Her serum creatinine value had been slowly increasing and she had been receiving treatment for hypertension since the age of 35 . She had a prior medical history of hysterectomy due to myoma uteri and a thyroidectomy due to papillary carcinoma, and had received thyroxin substitution. At referral, her blood pressure was $137 / 75 \mathrm{~mm} \mathrm{Hg}$ and her clinical status was normal. Her laboratory values were: serum creatinine, $145 \mu \mathrm{mol} / \mathrm{l}(1.9 \mathrm{mg} / \mathrm{dl})$; hemoglobin, $12.8 \mathrm{~g} / \mathrm{l}$; serum albumin, $42 \mathrm{~g} / \mathrm{l}$; calcium, $2.25 \mathrm{mmol} / \mathrm{l}$ (2.2-2.55); C-reactive protein, $1 \mathrm{mg} / \mathrm{l}(<5)$, and erythrocyte sedimentation rate, $9 \mathrm{~mm} / \mathrm{h}$. Standard immunological tests (ANA, ANCA, antiGBM, C3/C4, immunoelectrophoresis) as well as urine dip stick and urine microscopy were normal. Renal ultrasonography showed slightly reduced kidney size $(9.3$ and $9.1 \mathrm{~cm})$, and normal echogenicity. A renal angiogram showed no evidence of renal ar-
Einar Svarstad

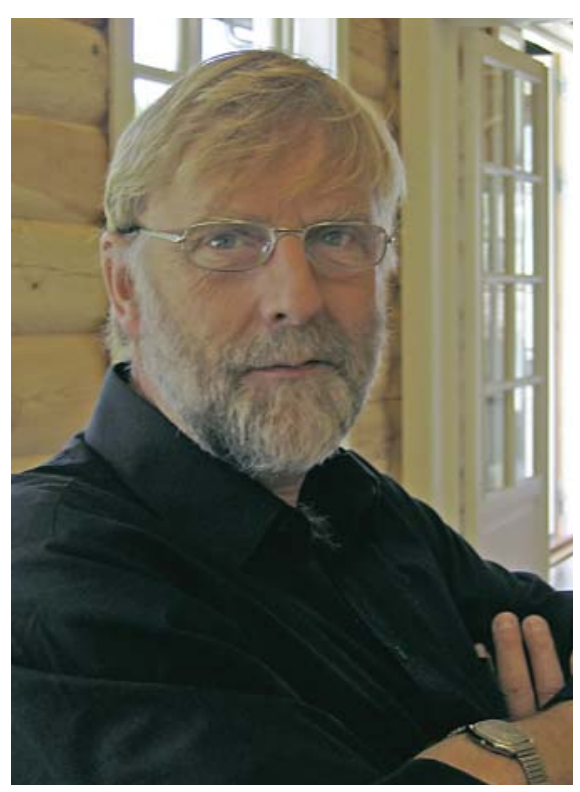

tery stenosis. A kidney biopsy was performed, and considered nondiagnostic, and a new biopsy was performed in 1994. During the following years, renal function gradually deteriorated and she underwent successful deceased donor kidney transplantation in 2007.

Patient 2, the daughter of patient 1, was referred to a nephrologist in 1999 at the age of 31. At that stage she had minimal proteinuria $(0.4 \mathrm{~g} / 24 \mathrm{~h})$, no hematuria, a normal serum creatinine

\section{KARGER}

Fax +41613061234 E-Mail karger@karger.ch www.karger.com (c) 2011 S. Karger AG, Basel

1660-2110/11/1191-0001\$38.00/0

Accessible online at:

www.karger.com/nec
Einar Svarstad

Institute of Medicine, University of Bergen

Haukeland University Hospital

NO-5021 Bergen (Norway)

Tel. +4755975000, E-Mail einar.svarstad@med.uib.no 

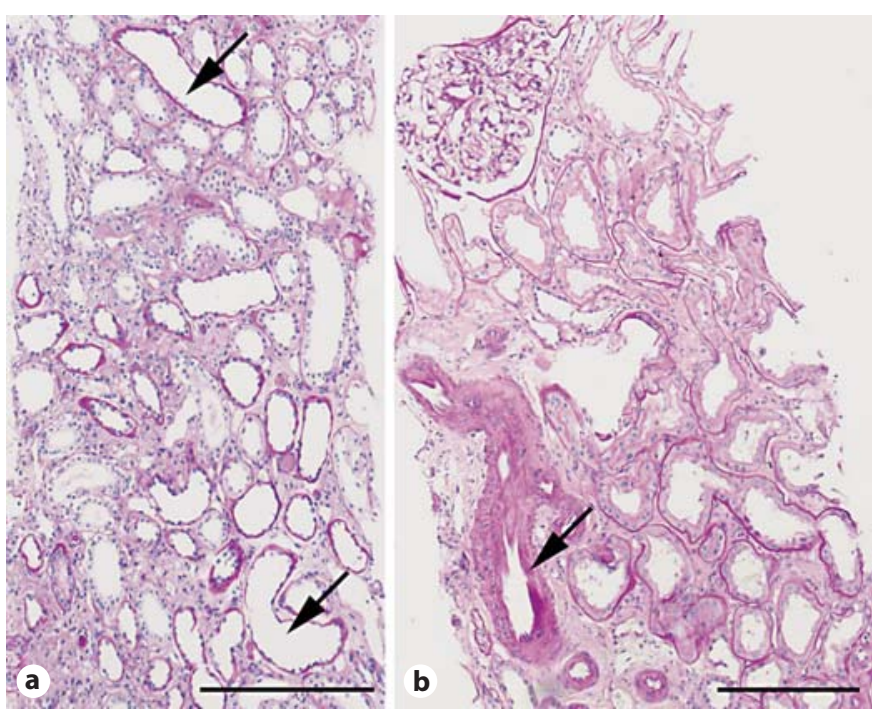

Fig. 1. Representative findings from the first (a) and the second biopsy (b) of the mother (patient 1). a Slightly widened atrophic tubules at the corticomedullary border (arrows). b Arteriosclerosis (arrow) and one normal glomerulus. Periodic acid-Schiff. Scale bars $=200 \mu \mathrm{m}$.
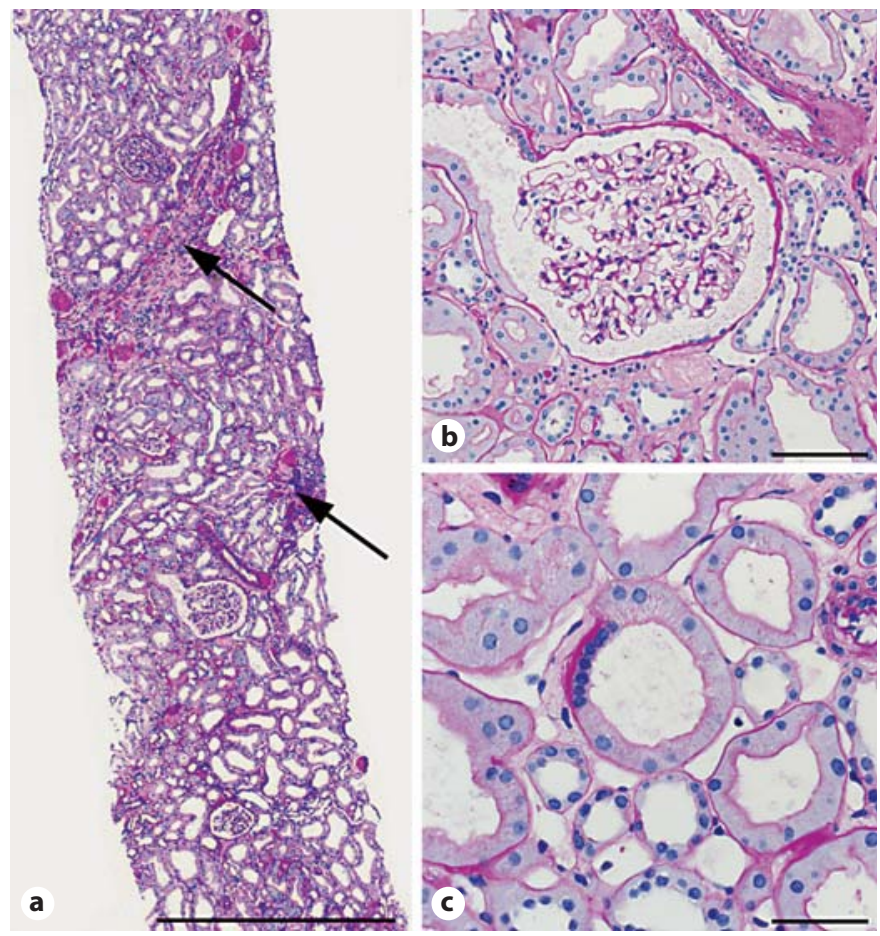

Fig. 2. Representative findings of the daughter's biopsy (patient 2). a Overview with foci with tubular atrophy and interstitial fibrosis (arrows). b An enlarged but otherwise normal glomerulus. c One of the few tubules with segmentally thickened basement membrane and abrupt transition from normal to dedifferentiated epithelium. Periodic acid-Schiff. Scale bars: $\mathbf{a}=1 \mathrm{~mm}, \mathbf{b}=100 \mu \mathrm{m}, \mathbf{c}=50 \mu \mathrm{m}$. value and her blood pressure was normal. Six years later she was re-referred due to increasing serum creatinine value and hypertension, for which she had been treated with an angiotensin receptor blocker since the age of 35 . Her past medical history was significant for repeated urinary tract infections, one episode of gout, and preeclampsia in two pregnancies. At referral, her blood pressure was 135/85 $\mathrm{mm} \mathrm{Hg}$ and the physical exam was normal. Laboratory values showed: serum creatinine, $120 \mu \mathrm{mol} / \mathrm{l}(1.57$ $\mathrm{mg} / \mathrm{dl})$; urea, $10.1 \mathrm{mmol} / \mathrm{l}(28.3 \mathrm{mg} / \mathrm{dl})$; uric acid, $525 \mu \mathrm{mol} / \mathrm{l}$ $(8.83 \mathrm{mg} / \mathrm{dl})$, and hemoglobin, $12.7 \mathrm{~g} / \mathrm{l}$. Urinary dip stick and urine microscopy were normal, but minimal microalbuminuria (albumin:creatinine ratio of $4 \mathrm{mg} / \mathrm{mmol}$ ) was observed. ANA, ANCA, immunoglobulins, complement $\mathrm{C} 3$ and $\mathrm{C} 4$, serum and urine electrophoresis were negative or normal. Renal ultrasound examination showed marginally normal-sized kidneys $(10.5 \mathrm{~cm}$ right, $10 \mathrm{~cm}$ left kidney), with reduced cortical thickness in the left kidney and normal echogenicity. A kidney biopsy was performed.

Dr. Leh: The first renal biopsy of the mother was taken at the age of 45 . The biopsy (fig. 1) showed arteriosclerosis and focal tubular atrophy, but was not representative since there was only one globally sclerosed glomerulus. At the corticomedullary border, there were some slightly widened tubules with thickened basement membranes, but there were no cysts. The second biopsy (fig. 1) of the mother 3 years later (at the age of 48) showed 4 glomeruli: one was globally sclerosed, the other glomeruli seemed normal. Again there was arteriosclerosis and focal tubular atrophy. The biopsy findings were unspecific and the descriptive diagnosis was benign nephrosclerosis.

Ten years later we examined the biopsy of the 37-year-old daughter (fig. 2). The biopsy contained 13 glomeruli, 6 of which were globally sclerosed. There were foci of tubular atrophy and interstitial fibrosis. The glomeruli looked normal, although some were enlarged. Only a couple of tubules showed segmentally thickened basement membranes and an abrupt transition from normal epithelium to dedifferentiated epithelium. Immunohistochemistry was negative. Electron microscopy showed thinning of the basement membranes in some capillary loops (fig. 3). The interpretation of the findings in the biopsy of the daughter was of moderate nephron loss of uncertain cause. The mother's biopsies were then re-examined, and electron microscopic investigation confirmed similar changes as in the daughter's biopsy with several capillary loops with thinned basement membranes (not shown).

\section{Discussion I}

Dr. Christiansen: A hereditary disease was suspected and the following diagnostic possibilities were considered. First, because of the finding of one or two tubuli with segmentally thickened basement membranes, we discussed whether this could be a disease related to the group of nephronophthisis/medullary cystic disease. However, no cysts were seen and the findings mentioned were minimal. Therefore, we felt this was speculative and likely not correct. In addition, nephronophthisis was re- 


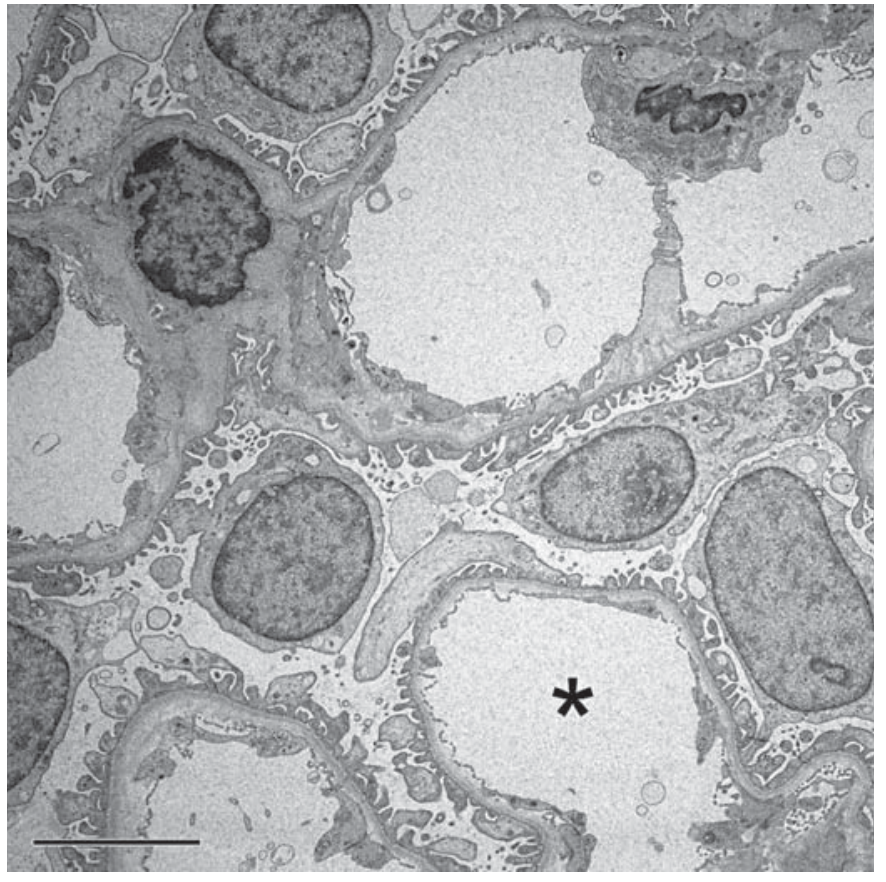

Fig. 3. Ultrastructure of the daughter's biopsy showing a glomerulus with capillary loops with thinned basement membranes $\left.{ }^{*}\right)$. There are no Alport-typical changes. The foot processes are preserved. Scale bar $=5 \mu \mathrm{m}$.

garded unlikely since this disease typically presents in childhood and shows an autosomal recessive pattern of inheritance. Second, regarding the thinning of the glomerular basement membranes in both patients, the question came up whether this could be a collagen IV nephropathy, thin basement membrane disease or a variant of Alport syndrome. However, the thinning was present in less than $50 \%$ of the glomerular capillaries and neither patient had hematuria, which rendered collagen IV disease an unlikely diagnosis.

\section{Questions and Answers}

Dr. Singh: There was segmental thinning of the glomerular basement membranes, but there were no Alporttypical changes, so at least the basement membrane changes would be more in line with the thin basement membrane abnormalities observed in thin basement membrane disease rather than in Alport's disease. In a prospective study of patients with thin basement membrane nephropathy, $13 \%$ initially had global glomerular sclerosis indicating nephron loss. In the follow-up, about
$30 \%$ became hypertensive and a few showed proteinuria. However, all patients had hematuria [1].

Dr. Fervenza: The thinning of the glomerular basement membranes suggested that this is a collagen IV nephropathy, maybe related to a mutation in the collagen IV alpha 3 or alpha 4 genes [2]. There is a series from Cyprus of 116 patients from 13 families with clinically diagnosed thin basement membrane nephropathy. [3]. Mutations in both collagen IV alpha 3 or alpha 4 genes were found in 82 patients from 10 families. In 20 patients, a dual diagnosis of thin basement membrane nephropathy and FSGS was made. During a follow-up of over 30 years, 31 of these 82 patients (37.8\%) developed chronic renal failure. More recently, the same group expanded their observations in 127 patients in 11 large families, where heterozygous mutations in collagen IV alpha 3 or alpha 4 genes were associated with hematuria, thin basement membrane nephropathy, late development of proteinuria, chronic renal failure, and ESRD due to FSGS [4]. Apparently there is a spectrum of diseases that can differ from the old-fashioned benign thin basement membrane or 'benign familial hematuria'. However, and different from the present case, all patients from the series from Cyprus have hematuria.

Dr. Singh: There is a lot of controversy around what criteria you use to make a diagnosis of thin basement membrane disease. Some people believe that less extensive thinning would also be acceptable to meet the diagnosis. The width of the basement membrane does not influence clinical presentation or outcome of thin glomerular basement membrane disease with persistent hematuria [5].

Dr. Leh: Our criteria are thinning of the glomerular basement membranes less than $250 \mathrm{~nm}$ in males and less than $220 \mathrm{~nm}$ in females, and at least $50 \%$ of the basement membranes should be involved [6]. We consider segmental thinning as a lesion of uncertain significance. However, in a study of 26 consecutive patients with hematuria, thin basement membranes, and no other glomerular pathology, the thinning of the basement membrane was segmental in about $30 \%$ of cases [7]. So there is no clear consensus about the criteria. When I was confronted with this family with a combination of partly unspecific, partly uncertain findings, I felt it right to suggest this differential diagnosis from a morphological point of view. Of course, learning that the patients did not have hematuria makes the diagnosis unlikely.

Dr. Singh: On the other hand, the patients do not have the features one normally would associate with nephronophthisis. There were no corticomedullary cysts and the fibrosis is rather focal than diffuse, as it is typical for nephronophthisis. 


\section{Discussion II}

Dr. Christiansen: We knew that our patients probably suffered from an unrecognized inherited nephropathy, but further diagnostic workup was halted for a long time until the patients provided information confirming that several family members were suffering from renal disease. Dr. Fiskerstrand will demonstrate the pedigree and explain the genetic investigations done.

Dr. Fiskerstrand: Looking at the pedigree (fig. 4), this fits with an autosomal dominant pattern of inheritance. So how could we identify the gene harboring a mutation and, thus, causing the disease in this Norwegian family? It could be a gene previously known to cause nephropathy, or we could be facing an unknown gene. We gathered blood samples from affected $(n=7)$ and unaffected $(n=$ 7) individuals (14 samples), purified DNA, and performed whole genome analysis with single-nucleotide polymorphisms (SNP) arrays. We used microarrays with 250,000 SNP markers. Linkage analysis (a specialized statistical analysis) was used on these marker data to identify a chromosomal region which was highly likely to harbor the mutation. In such a region, the affected have inherited the same (SNP) marker variants on one of their DNA strands (haplotypes), whereas the nonaffected have different haplotypes. The threshold for a significant linkage between a chromosomal region and the disease is set at a LOD score of 3 (linkage is 1,000 times more likely compared to nonlinkage).

Our linkage analysis identified a 3.3-Mb (i.e million base pairs) region on chromosome 16 with a LOD score of 2.3 (fig. 5). This was about the maximum LOD score we could achieve for any region in this family, due to the limited number of individuals analyzed. The candidate region contained a maximum of 57 genes and among these was $U M O D$, encoding uromodulin (Tamm-Horsfall protein). This is a moderately sized gene of 11 exons and mutations in this gene were known to cause nephropathy. Uromodulin is the most common protein in the urine, excreted by cleavage of the GPI anchor in this transmembrane glycoprotein located in the thick ascending limb of the loop of Henle.

By sequencing, we identified heterozygosity for a novel missense mutation in $U M O D$ in all affected individuals (fig. 6). In position 800 , there was a base exchange (c. $800 \mathrm{G}>\mathrm{T}$ ) which theoretically led to the replacement of the amino acid cysteine with phenylalanine in the protein in codon 267 (p.Cys267Phe). Nearly all mutations in the UMOD gene are missense. Moreover, about two thirds of these involve a cysteine residue. Thus, the mu-

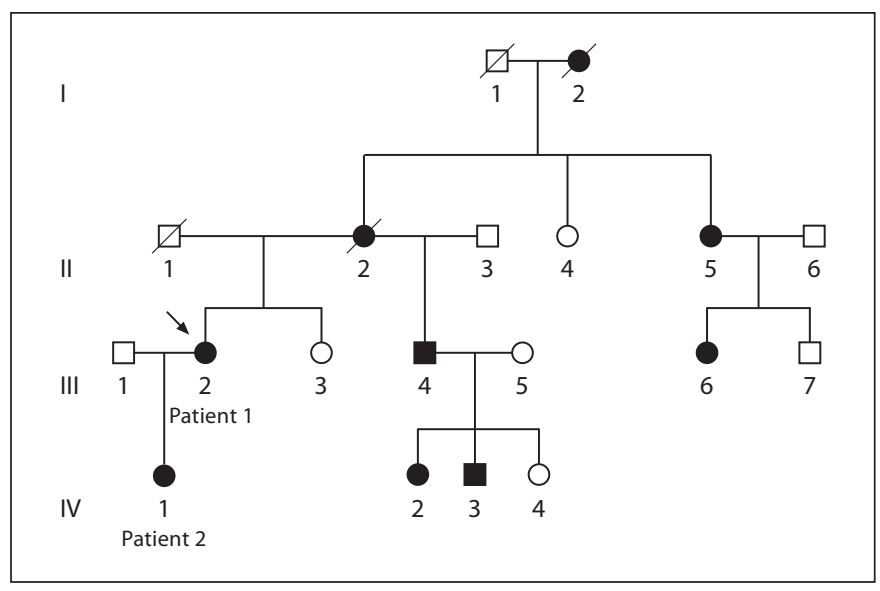

Fig. 4. Pedigree showing the affected mother (patient 1) and daughter (patient 2) and their relatives. Individuals with kidney failure are shown in black.

tation found in the affected members in this family is highly likely to be pathogenic, and compatible with the diagnosis of uromodulin-related nephropathy. The present mutation sits in a cysteine-rich D8C domain which is important for correct folding of the protein. According to Williams et al. [8], mutant uromodulin may be retained in the endoplasmic reticulum and cellular trafficking is delayed, leading to reduced uromodulin secretion into urine. The protein might undergo degradation via the proteosomal or autophagic pathways. Uromodulin is expressed in cilia and therefore UMOD mutation is a form of ciliopathy [9].

Dr. Leh: Having found a mutation in the UMOD gene and knowing that uromodulin is produced in the straight part of the distal tubules, i.e. the thick ascending limb of the loop of Henle, we undertook a re-examination of the biopsies with special focus on the distal tubules. The epithelial cells showed weakly stained inclusions (fig. 7a). These inclusions are well demarcated in an immunhistochemical stain for cytokeratin (fig. 7b). The inclusions can also be seen in the semi-thin sections for electron microscopy (fig. 7c). The inclusions are moderately electrondense in the ultrastucture (fig. 8a). In addition, many distal tubular epithelial cells show densely packed lamellae representing hyperplastic endoplasmic reticulum (fig. 8a, b). Some cisternae are dilated and contain amorphous material (fig. 8b). Of note, the tertiary structure of uromodulin might be changed because of the mutation. The inclusions and the dilated cisternae represent accumulated uromodulin. According to this, both immunohistochemistry and immunofluorescence show intensely 


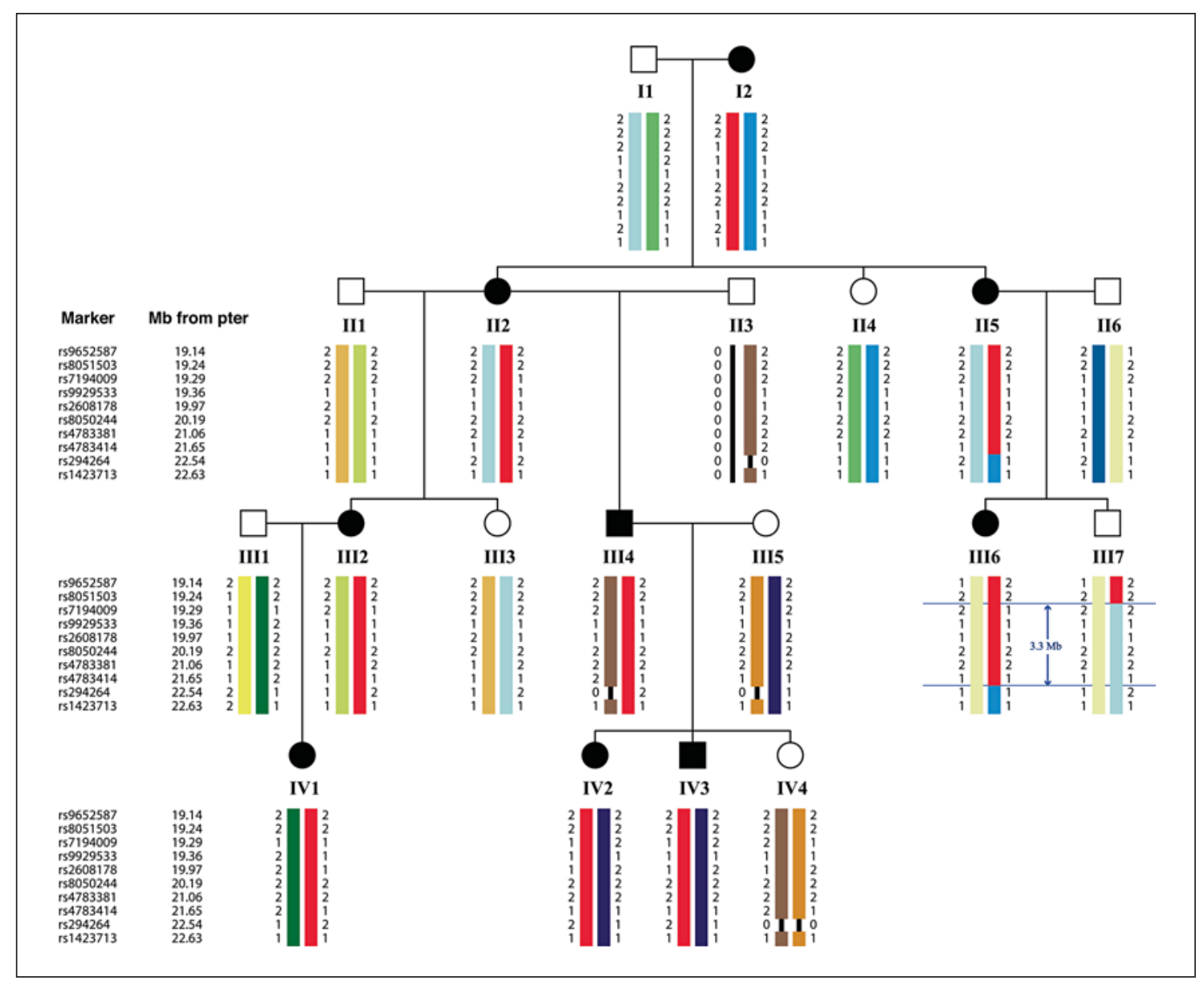

Fig. 5. Haplotypes in the candidate region on chromosome 16, shown in the pedigree. SNP markers are displayed in the region 19.14-22.63 Mb from the end of the short arm on chromosome 16 (pter). All of the affected share whole or part of the same DNA strand in this region (red color), inherited from individual I2. In individual II5 (affected), a crossing over between the two maternal strands can be observed, limiting the candidate region for the mutation in the distal direction to rs294264 (22.54 Mb from pter).
Likewise, her healthy son (III7) also has a crossing over and shares part of the red haplotype with the affected, thus limiting the candidate region proximally to the marker rs 8051503 (19.24 Mb from pter). The candidate region between rs 8051503 and rs294264 is ca. $3.3 \mathrm{Mb}$ and contains about 57 genes. Samples from all individuals shown, except I1, I2, II1, II2, and II3, were included in the linkage analysis using SNP-arrays (50K). positive accumulations of uromodulin in distal tubular epithelial cells compared to normal controls (fig. 9). In conclusion, the biopsy changes in the distal tubules of the mother and daughter are in line with a previous report by Nasr et al. [10] and compatible with the diagnosis of a UMOD mutation.

Dr. Christiansen: As we have shown, this family has uromodulin-associated kidney disease. UMOD mutations show an autosomal dominant inheritance pattern and the main phenotypic expressions are medullary cystic kidney disease type II and familial hyperuricemic nephropathy; both phenotypes were seen in our family. It is important to recognize that hyperuricemia is present in the majority of individuals with UMOD-related kidney disease and occurs independently of the development of kidney failure [11]. However, gout is recorded in only $45 \%$ of individuals with a UMOD mutation, with onset of gout ranging from age 8 to 38 years [12]. Gout tends to worsen and the frequency of attacks increases deterioration of kidney function.

Patients with medullary cystic kidney disease type II typically develop renal failure at $40-70$ years of age and seldom have gout, while patients with familial juvenile hyperuricemic nephropathy usually develop renal failure 
Fig. 6. Upper panel shows the normal sequence and lower panel shows the sequence in one of the affected individuals in exon 3 in the UMOD gene. In position 800 in the coding sequence, the affected are heterozygous for a novel base exchange (C>T, marked by an arrow). This theoretically leads to the replacement of the amino acid cysteine with phenylalanine in position 267 in the protein (p.Cys267Phe).

Fig. 7. Distal tubules from the daughter's biopsy with inclusions. a The inclusions are weakly stained by periodic acid-Schiff stain (arrows). b Immunohistochemistry for cytokeratin (AE1/AE3) stains the tubular epithelial cells intensely but leaves the inclusions (arrows) unstained. c Tubular inclusions (arrow) in a semi-thin section for electron microscopy. Scale bars $=20$ $\mu \mathrm{m}$.

Fig. 8. Ultrastructure of distal tubules. a Distal tubular epithelial cells with moderately electron-dense inclusions (arrows) and hyperplastic endoplasmic reticulum $\left.{ }^{*}\right)$. b Lamellae of hyperplastic endoplasmic reticulum with dilated cisternae (arrows), containing moderately electrondense material.

\section{GGTGAAGGCCTGTGCCGGCGGCTA}

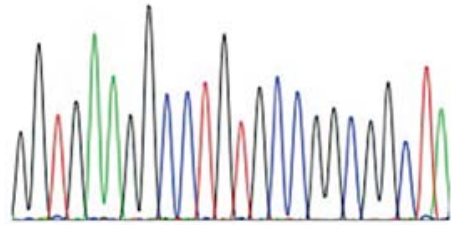

Wild-type

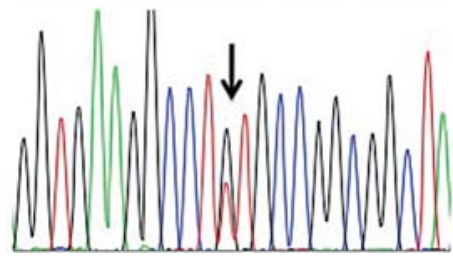

c.800G $>\mathrm{T}$ (heterozygous) p.Cys267Phe
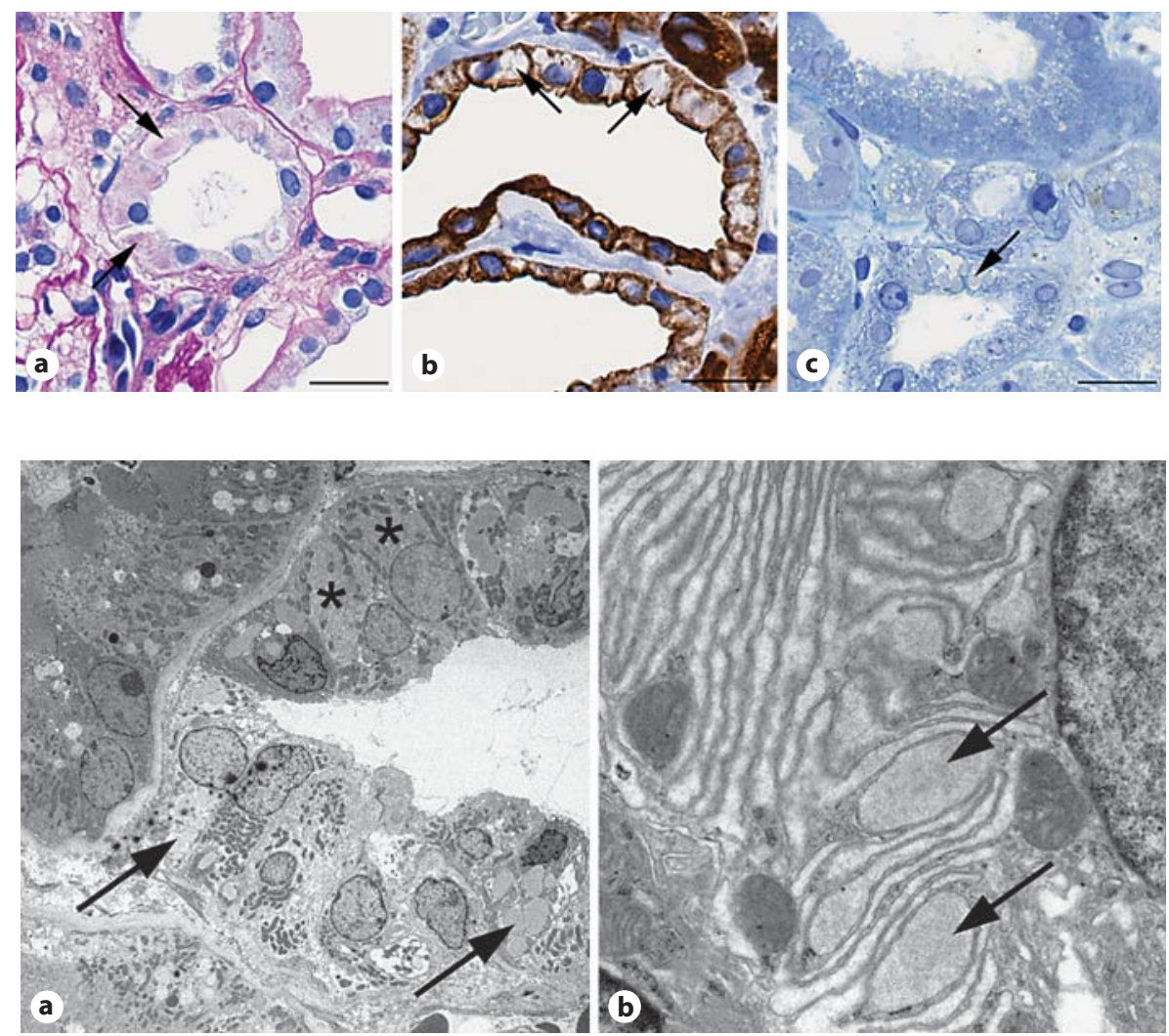

earlier (at 30-40 years of age) and frequently present with gout and hyperuricemia. There seems to be a consensus now that these presentations are variants of the same disease [13].

Although uromodulin is the most abundant protein in urine, its biological function is unclear. It has been implicated in binding and excretion of injurious products from tubular fluids as well as in protection against infections. On the other hand, interstitial accumulation seems to be associated with inflammation and progressive scarring in some patients with chronic kidney disease (CKD) [14]. $U M O D$ mutations lead to decreased urinary levels of uromodulin and possibly aggregation of uromodulin in tubular epithelium. 
Fig. 9. Immunohistochemistry $(\mathbf{a}, \mathbf{b})$ and immunofluorescence (c, d; uromodulin green, acetylated $\alpha$-tubulin red, nuclei blue; confocal laser scanning microscopy) for uromodulin in normal distal tubules (a, c) and distal tubules from the daughter's biopsy (b, d). Normal distal tubules show diffuse cytoplasmic staining with luminal accentuation. In uromodulin-associated kidney disease, uromodulin is accumulated in clusters in the cytoplasm. Scale bars $\mathbf{a}$ and $\mathbf{b}=20 \mu \mathrm{m}, \mathbf{c}$ and $\mathbf{d}=10 \mu \mathrm{m}$.
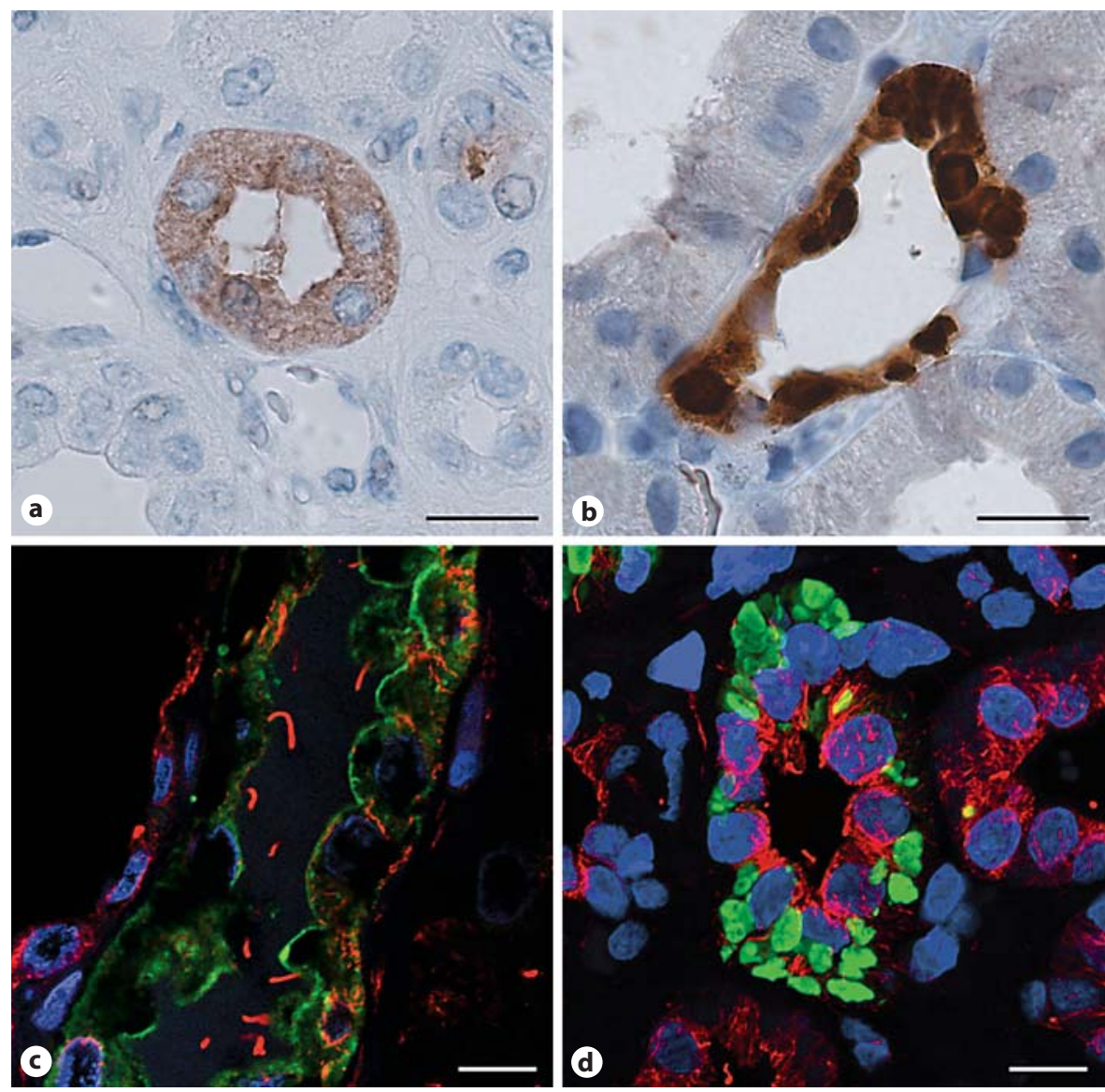

\section{Questions and Answers}

Dr. Fervenza: This is a most interesting case. Does the lesion in the distal tubule cause any urinary concentration abnormalities or renal acidosis?

Dr. Christiansen: We do not have any evidence of that, and no acidosis was demonstrated in our patients.

Dr. Fervenza: How do you put together the uromodulin mutation with the presence of those thin basement membranes?

Dr. Leh: I have no good explanation for the thin glomerular basement membrane in these patients. The glomeruli in the daughter were enlarged; one might speculate that this could lead to some distension of the capillary walls and, thus, to thinning of the glomerular basement membrane [15].

Dr. Singh: A number of conditions are associated with thinning of the glomerular basement membrane, e.g. IgA nephropathy and lupus nephritis [16], and might imply some impairment of glomerular basement membrane synthesis that may arise for a variety of reasons. Thus, the presence of these changes could constitute a nonspecific finding. These cases demonstrate an interesting journey of discovery which included a morphological and genetic diagnosis. There are a few comments I would like to make. There is a convergence of studies now suggesting that uromodulin is an important factor in the development of CKD, perhaps of unknown identity. In fact, Köttgen et al. [17], using a genome-wide association study, recently reported on a link between uromodulin and CKD. A metaanalysis of genome-wide association data on about 60,000 individuals with CKD was performed, demonstrating a significant association between uromodulin and susceptibility to CKD. So it seems that the UMOD gene may be associated with significant kidney disease and this case might somewhat illustrate this.

Dr. Fervenza: As mentioned by Dr. Singh, genomewide association studies have been used to investigate genetic components of phenotypes that do not exhibit classical Mendelial inheritance as a result of single gene mutation. This approach has led to identification in many parts of the human genome associated with complex 
traits such as diabetes and hypertension $[18,19]$. An association of variants at UMOD with chronic kidney disease and kidney stones has also been reported using this approach [20]. However, genome-wide association studies require DNA samples from a large number of individuals with or without a demonstrable phenotype in order to provide adequate statistical power to identify SNPs that are associated with a particular trait.

Dr. Fervenza: I am a bit curious as to why the patients get hyperuricemia. Are the cilia somehow involved in uric handling and reabsorption?

Dr. Leh: Due to the UMOD mutation, the sodium reabsorption in the distal tubules is disturbed and urine concentration capacity is reduced. According to the same mechanism as in long-term administration of loop diuretics, sodium uptake in proximal tubules is increased and is accompanied by increased urate reabsorption [21]

Dr. Singh: Considering the management of these patients, how did you deal with genetic counseling?

Dr. Fiskerstrand: We offered genetic counseling to all affected individuals before the genetic analysis and again after the mutation was found.

Dr. Singh: There is now a possibility for uromodulin staining by immunohistochemistry/immunofluorescence. Would it be possible to stain, for example, nephrosclerosis cases retrospectively and pick up the cases without even having to do more careful genetic studies?

Dr. Leh: This is a very good idea. It would be interesting to look at biopsies from patients with unexplained CKD to see whether there is accumulation of uromodulin.

Dr. Singh: We have this large diagnostic basket labeled 'benign nephrosclerosis' - some of them are not even biopsied. This opens up the possibility of further diagnostic identification with biopsy, immunofluorescence, and genome studies. It opens up new diagnostic possibilities.

Dr. Fiskerstrand: There are also exciting new possibilities for genetic diagnostics with the new whole genome 'deep' sequencing technique, which soon may be used in diagnostic routine, at least exome sequencing where you just sequence all the exons (coding parts of the genes). Then, if you have a case of, for example, unexplained possibly inherited nephropathy, you may run an exome sequencing to see if there are mutations in any known disease genes, or if you accidentally find a mutation in new possible candidate genes for this type of disease.

Dr. Svarstad: Another consequence of these new techniques would of course be that some familiar renal diseases may be correctly diagnosed without the need of a renal biopsy. In some of these cases, the biopsy indication may shift from a diagnostic to a more prognostic or staging purpose.

Dr. Singh: I am again trying to simulate the action of cilia. Some people in the field of polycystic kidney disease (PKD) firmly believe that the cilia modify the PKD phenotype, and in this particular case the cilia may be involved not in cysts but in distal tubules. What is notable is that in some of these diseases, such as UMOD mutations, you see corticomedullary cysts, not the widespread cysts as in PKD, suggesting that either this is not such a profound disease of the cilia or that PKD reflects simply more than abnormalities in the cilia. We seem to develop a cadre of diseases with abnormalities in cilia function and structure.

Dr. Fervenza: Why do the patients develop renal failure?

Dr. Leh: Mutated uromodulin is accumulated in distal tubular cells. The hypothesis is that these cells go into apoptosis which triggers inflammation and fibrosis and subsequent renal failure [10,22].

Dr. Svarstad: Is it possible to measure uromodulin excretion in the urine to distinguish this type of hereditary nephropathy?

Dr. Fervenza/Dr. Singh: We do not measure uromodulin excretion at Harvard or the Mayo Clinic. We do not think the awareness at this stage is high among clinicians for considering the possibility of UMOD-associated disease and you probably also need more studies on the predictive value of uromodulin excretion in relation to diagnosis of this condition.

\section{Conclusion}

Uromodulin-associated kidney disease is a hitherto rare and probably underdiagnosed disease. Patients with this disorder may show few symptoms and findings except hypertension, slowly progressive kidney failure, and gout. The diagnostics of rare inherited kidney diseases is a multidisciplinary challenge, and genetic work-up should be introduced at an early stage. Our cases demonstrate the use of linkage analysis using SNP microarrays in the diagnosis of a novel missense-mutation in UMOD. New powerful techniques like exome sequencing are rapidly emerging and probably have the capacity to identify a substantial amount of hitherto unexplained hereditary renal diseases. Generally, many laboratories offering linkage analysis would be interested in potential collaborative programs on a research basis, and thereby allow access to this technique to countries where this technology is not available. 


\section{References}

$\checkmark 1$ Nieuwhof CM, de Heer F, de Leeuw P, van Breda Vriesman PJ: Thin GBM nephropathy: premature glomerular obsolescence is associated with hypertension and late onset renal failure. Kidney Int 1997;51:1596-1601.

$\checkmark 2$ Kashtan CE: Familial hematuria. Pediatr Nephrol 2009;24:1951-1958.

3 Voskarides K, Damianou L, Neocleous V, Zouvani I, Christodoulidou S, Hadjiconstantinou V, Ioannou K, Athanasiou Y, Patsias C, Alexopoulus E, Pierides A, Kyriacou K, Deltas C: COL4A3/COL4A4 mutations producing focal segmental glomerulosclerosis and renal failure in thin basement membrane nephropathy. J Am Soc Nephrol 2007; 18:3004-3016.

$\checkmark 4$ Pierides A, Voskarides K, Athanasiou Y, Ioannou K, Damianou L, Arsali M, Zavros M, Pierides M, Vargemezis V, Patsias C, Zouvani I, Elia A, Kyriacou K, Deltas C: Clinicopathological correlations in 127 patients in 11 large pedigrees, segregating one of three heterozygous mutations in the COL4A3/ COL4A4 genes associated with familial haematuria and significant late progression to proteinuria and chronic kidney disease from focal segmental glomerulosclerosis. Nephrol Dial Transplant 2009;24:2721-2729.

$\checkmark 5$ Szeto CC, Mac-Moune Lai F, Kwan BC, Leung CB, Choi PC, Pang WF, Chow KM, Lai, KB, Wang G, Li PK: The width of the basement membrane does not influence clinical presentation or outcome of thin glomerular basement membrane disease with persistent hematuria. Kidney Int 2010;78: 1041-1046.

-6 Foster K, Markowitz GS, D‘Agati VD: Pathology of thin basement membrane nephropathy. Semin Nephrol 2005;25:149-158.

$\checkmark 7$ Ivanyi B, Pap R, Ondrik Z: Thin basement membrane nephropathy: diffuse and segmental types. Arch Pathol Lab Med 2006; 130:1533-1537.
8 Williams SE, Reed AA, Galvanovskis J, Antignac C, Goodship T, Karet FE, Kotanko P Lhotta K, Morinière V, Williams $\mathrm{P}$, Wong W, Rorsman P, Thakker RV: Uromodulin mutations causing familial juvenile hyperuricaemic nephropathy lead to protein maturation defects and retention in the endoplasmatic reticulum. Hum Mol Genet 2009;18:29632974.

-9 Zaucke F, Boehnlein JM, Steffens S, Polishchuk RS, Rampoldi L, Fischer A, Pasch A, Boehm CW, Baasner A, Attanasio M, Hoppe B, Hopfer H, Beck BB, Sayer JA, Hildebrandt F, Wolf MT: Uromodulin is expressed in renal primary cilia and UMOD mutations result in decreased ciliary uromodulin expression. Hum Mol Genet 2010;19:1985-1997.

10 Nasr SH, Lucia JP, Galgano SJ, Markowitcz GS, D‘Agati VD: Uromodulin storage disease. Kidney Int 2008;73:971-976.

11 Bleyer AJ, Zivná M, Kmoch S: Uromodulinassociated kidney disease. Nephron Clin Pract 2011;118:c31-c36.

12 Dahan K, Devuyst O, Smaers M, Vertommen D, Loute G, Poux JM, Viron B, Jacquot C, Gagnadoux MF, Chauveau D, Buchler M, Cochat P, Cosyns JP, Mougenot B, Rider MH, Antignac C, Verellen-Dumoulin C, Pirson Y: A cluster of mutations in the UMOD gene causes familial juvenile hyperuricemic nephropathy with abnormal expression of uromodulin. J Am Soc Nephrol 2003;14:28832893.

13 Kumar S: Mechanism of injury in uromodulin-associated kidney disease. J Am Soc Nephrol 2007;18:10-12.
14 Prajczer S, Heidenreich U, Pfaller W, Kotanko P, Lhotta K, Jennings P: Evidence for a role of uromodulin in chronic kidney disease progression. Nephrol Dial Transplant 2010; 25:1896-1903.

15 Hill GS, Jenis EH, Goodloe S Jr: The nonspecificity of the ultrastructural alterations in hereditary nephritis with additional observations on benign familial hematuria. Lab Invest 1974;31:516-532.

16 Norby SM, Cosio FG: Thin basement membrane nephropathy associated with other glomerular diseases. Semin Nephrol 2005; 25:176-179.

17 Köttgen A, Pattaro C, Böger CA, Fuchsberger $\mathrm{C}$, Olden $\mathrm{M}$, et al: New loci associated with kidney function and chronic kidney disease. Nat Genet 2010;42:376-384

18 Adeyemo G, Gerry N, Chen G, et al: A genome-wide association study of hypertension and blood pressure in African Americans. PLoS Genet 2009; 5:e1000564.

$>19$ Billings LK, Florez JC: The genetics of type 2 diabetes: what have we learned from GWAS? Ann NY Acad Sci 2010;1212:59-77.

20 Gudbjartsson DF, Holm H, Indridason OS, et al: Association of variants at UMOD with chronic kidney disease and kidney stones - role of age and comorbid diseases. PLoS Genet 2010;6(11)

21 Scolari F, Caridi G, Rampoldi L, Tardanico R, Izzi C, Pirulli D, Amoroso A, Casari G, Ghiggeri GM: Uromodulin storage diseases: clinical aspects and mechanisms. Am J Kidney Dis 2004;44:987-999.

22 Choi SW, Ryu OH, Choi SJ, Song IS, Bleyer AJ, Hart TC: Mutant Tamm-Horsfall glycoprotein accumulation in endoplasmic reticulum induces apoptosis reversed by colchicine and sodium 4-phenylbutyrate. J Am Soc Nephrol 2005;16:3006-3014. 